\title{
Estudo da sinterização em reator vertical de leito fixo para produção de agregado sintético
}

\author{
(Sintering study in vertical fixed bed reactor for \\ synthetic aggregate production) \\ D.S.Quaresma ${ }^{1}$,A.S.S. Neves ${ }^{1}$, A.O. Melo ${ }^{1}$,L.F.S.Pereira ${ }^{2}$, \\ P.T.S.Bezerra ${ }^{3}$ E. N.Macêdo ${ }^{l}$, J.A.S.Souza ${ }^{1}$ \\ ${ }^{1}$ Programa de Pós-Graduação em Engenharia de Recursos Naturais da Amazônia; \\ ${ }^{2}$ Programa de Pós-Graduação em Engenharia Química; ${ }^{3}$ Faculdade de Engenharia Química, \\ Universidade Federal do Pará, R. Augusto Corrêa, 1, Belém, PA 66075-110 \\ danysq@gmail.com
}

\begin{abstract}
Resumo
Os agregados sintéticos vêm sendo empregados na construção civil como uma medida para redução do extrativismo mineral. Dentro desse contexto, a reciclagem de resíduos industriais configura hoje a base da maioria dos processos de redução da exploração dos recursos minerais. Neste trabalho estudou-se a sinterização em reator vertical de leito fixo para produção de agregado sintético, a partir de $20 \%$ de pelotas e $80 \%$ de carvão vegetal. As pelotas foram confeccionadas a partir de uma mistura contendo argila, carvão vegetal e cinza volante. Realizaram-se dois experimentos variando a velocidade de sucção do ar. O material produzido foi analisado por difração de raios X, microscopia eletrônica de varredura, medidas de suas propriedades cerâmicas e classificação quanto à sua granulometria. As análises dos resultados demonstraram que as reações de estado sólido, durante o processo de sinterização, foram eficientes e que o agregado produzido foi classificado como leve e graúdo. O processo se mostrou interessante para a sinterização de agregados, podendo ser controlado pela composição, granulometria, gradiente de temperatura e fluxo gasoso.
\end{abstract}

Palavras-chave: cinza volante, agregado sintético, sinterização, reator de leito fixo.

\begin{abstract}
The synthetic aggregates are being employed in civil construction for the reduction of mineral extraction activities. Within this context, the recycling of industrial waste is the basis of the majority of processes to reduce the exploitation of mineral resources. In this work the sintering in a vertical fixed bed reactor for synthetic aggregate production using $20 \%$ pellets and $80 \%$ charcoal was studied. The pellets were prepared from a mixture containing clay, charcoal and fly ash. Two experiments varying the speed of air sucking were carried out. The material produced was analyzed by X-ray diffraction, scanning electron microscopy, measures of their ceramic properties, and particle size analysis. The results showed that the solid-state reactions, during the sintering process, were efficient and the produced material was classified as coarse lightweight aggregate. The process is interesting for the sintering of aggregates, and can be controlled by composition, particle size, temperature gradient and gaseous flow.
\end{abstract}

Keywords: fly ash, synthetic aggregate, sintering, fixed bed reactor.

\section{INTRODUÇÃO}

A sociedade moderna valoriza processos que envolvam reciclagem de materiais. Deste modo, os investimentos relacionados ao aproveitamento de resíduos tornaram-se essenciais ao desenvolvimento como alternativa de proteção ambiental [1]. Um exemplo disto são as usinas termelétricas, que geram cinzas a partir da queima de carvão mineral. Este resíduo vem sendo estudado visando, entre outras aplicações, um possível aproveitamento como matéria-prima na fabricação de blocos e tijolos além de produtos cerâmicos especiais [2,3]. As cinzas volantes como matéria-prima para produção de agregado sintético apresentam-se como outra alternativa para o reaproveitamento do resíduo em função das características físico-químicas que este material apresenta.
As características físicas, químicas e mineralógicas das cinzas dependem de fatores como a composição do carvão de origem, as condições de combustão, o tipo e a eficiência do sistema de controle de emissões e os métodos de disposição utilizados [4]. Em temperaturas adequadas, este material favorece a formação de fases mineralógicas que propiciam um aumento da resistência mecânica do agregado produzido, como é o caso da mulita. Essas características são normalmente obtidas através da queima deste material em temperaturas superiores a $1100{ }^{\circ} \mathrm{C}$. A qualidade do produto acabado depende, fundamentalmente, da matériaprima utilizada, da temperatura de queima e do processo de conformação da massa cerâmica [5].

Segundo dados estatísticos, no Brasil são gerados aproximadamente $1,7 \times 10^{6}$ ton de cinzas de carvão mineral 
por ano; apesar de quimicamente inerte, este resíduo apresenta problemas ambientais pela sua constituição granulométrica e pela quantidade gerada [6]. Em relação à combustão em meio poroso utilizando reator de leito fixo, a fase sólida reage com a fase gasosa, onde ocorrem reações químicas como a pirólise, a combustão do residual de carbono e as reações de estado sólido. Estas reações de transformação são acompanhadas por mudanças bruscas na estrutura física da fase sólida [7]. Diante deste cenário, o presente trabalho propôs produzir agregado sintético a partir de resíduo industrial, cinza volante, utilizando-a em uma mistura de argila e carvão vegetal (biomassa), por meio de sinterização em reator vertical de leito fixo.

\section{MATERIAIS E MÉTODOS}

$\mathrm{Na}$ elaboração deste trabalho foram utilizadas como matérias-primas, a cinza volante de carvão mineral, argila e carvão vegetal. A cinza volante foi cedida pela refinaria de alumina Hydro Alunorte, localizada no município de Barcarena no estado do Pará e trata-se de um rejeito do processo de queima do carvão mineral em caldeiras de leito fluidizado circulante empregada na produção de energia. A argila é do tipo caulinítica, proveniente das margens do Rio Guamá no entorno da Universidade Federal do Pará e o carvão vegetal foi obtido comercialmente. A cinza, resíduo industrial alvo desta pesquisa, não necessitou de nenhum tratamento prévio referente à cominuição para desagregação de suas partículas por ser constituída por partículas de granulometria fina. Assim foi utilizada de forma direta nos ensaios experimentais.

A argila por apresentar plasticidade proporciona, quando umedecida com água, boa trabalhabilidade ao material. A argila passou por um processo de secagem a $105^{\circ} \mathrm{C} \mathrm{em}$ estufa com recirculação de ar durante 24 h e posteriormente foi desagregada em moinho de bolas por $30 \mathrm{~min}$. O carvão vegetal funciona como combustível sólido no processo de sinterização e foi utilizado nos ensaios experimentais tanto na mistura de composição das pelotas quanto no preenchimento do leito do reator de sinterização (correspondendo a $80 \%$ do volume do reator) para a produção do agregado sintético. No âmbito da mistura de composição das pelotas o carvão passou por secagem a $105^{\circ} \mathrm{C}$, em seguida foi britado em britador de mandíbulas e posteriormente moído em moinho de bolas até que sua granulometria fosse inferior a 100 mesh $(0,149 \mathrm{~mm})$.

As matérias-primas processadas foram caracterizadas quanto à sua distribuição granulométrica, segundo a normatização brasileira [8]. Os materiais submetidos à fluorescência de raios $\mathrm{X}$ e difração de raios $\mathrm{X}$ foram

Tabela I - Velocidade de sucção do ar. [Table I - Air suction speed.]

\begin{tabular}{cc}
\hline Experimento & $\mathrm{V}_{\mathrm{AR}}\left(\mathrm{m} \cdot \mathrm{s}^{-1}\right)$ \\
\hline 1 & 0,68 \\
2 & 1,26 \\
\hline
\end{tabular}

pulverizados e classificados para uma granulometria abaixo de 200 mesh $(0,074 \mathrm{~mm})$ em peneira Tyler. O sistema difratométrico utilizado foi o Empyrean com material anódico de cobalto e os resultados interpretados no programa X'Pert HighScore. No carvão de forma específica realizou-se sua análise imediata segundo a normatização brasileira [9].

Fabricação do agregado sintético: para a obtenção do agregado sinterizou-se uma mistura com $20 \%$ do volume do reator com pelotas (constituída a partir das matérias-primas) e $80 \%$ com carvão vegetal (quantidade suficiente para sinterizar as pelotas). Foram realizados dois experimentos para a obtenção do agregado sintético variando-se a velocidade de sucção do ar contendo o comburente das reações de combustão, que promoveram a sinterização ao longo do reator (Tabela I). A escolha das velocidades de sucção foi estabelecida a partir de testes anteriores, com o objetivo de obter temperaturas mais elevadas [10].

Fabricação das pelotas e do carvão vegetal: para a preparação das pelotas, foi realizada uma mistura previamente seca de $40 \%$ de cinza volante, $30 \%$ de argila e $30 \%$ de carvão vegetal em massa. A homogeneização foi feita em moinho de bolas durante $1 \mathrm{~h}$. Após essa etapa, a mistura foi colocada em um tambor rotativo onde as pelotas foram formadas com adição de $40 \%$ de água em massa. As pelotas produzidas apresentaram tamanhos variados, conforme mostra a Fig. 1. Posteriormente, o material passou por processo de secagem durante $24 \mathrm{~h}$. As pelotas foram classificadas para uma granulometria no intervalo entre 6,35 e $4 \mathrm{~mm}$ e foram avaliadas quanto à sua distribuição granulométrica e densidade aparente. O carvão utilizado para preencher $80 \%$ do volume do reator, passou por secagem e britagem para, em seguida, ser triturado em moinho de discos e classificado para uma granulometria no intervalo entre 3,36 e 1,70 mm. A análise granulométrica e densidade aparente foram realizadas segundo as normas brasileiras [8, 11]. Após a classificação, análises, medição dos percentuais em volume e massa das pelotas e do carvão, esses materiais foram homogeneizados manualmente e caracterizados quanto à sua densidade aparente. A mistura foi submetida a processo de secagem por $24 \mathrm{~h}$ a $105^{\circ} \mathrm{C}$, em estufa com recirculação de ar. Em seguida, a mistura foi sinterizada em reator de leito fixo.

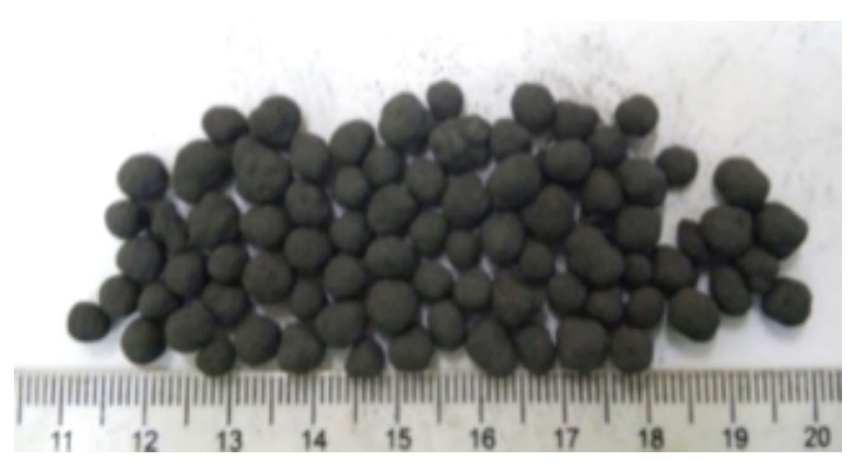

Figura 1: Pelotas formadas em tambor rotativo. [Figure 1: Pellets formed in a rotary drum.] 
Dispositivo experimental: o dispositivo experimental construído para a obtenção do agregado sintético consistiu em um reator de leito fixo (Fig. 2) com base em um trabalho desenvolvido na Universidade de Toulouse [7]. $\mathrm{O}$ equipamento foi projetado para permitir um processo de combustão em cocorrente unidimensional, sendo constituído por uma câmara de combustão cilíndrica vertical de $158 \mathrm{~mm}$ de diâmetro interno e uma altura de $700 \mathrm{~mm}$. O diâmetro foi escolhido para ser grande o suficiente para limitar as perdas de calor através das paredes, mas também estreito o suficiente para evitar o uso de grandes quantidades de material a ser sinterizado. O leito fixo foi produzido em aço carbono de 3,2 $\mathrm{mm}$ de espessura, entre a câmara de combustão e o cilindro vertical externo de $164 \mathrm{~mm}$ de diâmetro. Foi adicionada lã de rocha com a finalidade de ser utilizada como isolante térmico. Uma grelha de aço carbono foi localizada na parte inferior da câmara, apoiada por um tubo interno. Ao longo da região vertical do reator, sete termopares foram introduzidos nos furos localizados em diferentes alturas para medir a temperatura ao longo do eixo da célula de combustão. Um termopar foi adicionado para medir a temperatura da parede do cilindro externo e outro localizado no tubo interno na região inferior ao reator para medir a temperatura dos gases de combustão.

Protocolo dos experimentos: os experimentos iniciaram com à preparação do dispositivo experimental (Fig. 3). Uma grelha de aço carbono foi introduzida na parte inferior da câmara de combustão do reator e posteriormente acoplada a um tubo de aço zincado de $100 \mathrm{~mm}$ de diâmetro, conectado a um exaustor para succionar o ar que continha o comburente da reação além de transportar os gases de combustão. Em seguida, os termopares foram colocados em diferentes regiões do reator e na saída do mesmo. Próximo ao termopar utilizado para medir a temperatura dos gases de combustão, conectou-se um manômetro para constatar a passagem do fluxo de ar no início do experimento. A aquisição dos dados de temperatura dos termopares acoplados ao reator de leito fixo foi registrada no Paperless Recorder do tipo PHL, a cada $5 \mathrm{~s}$. Após a coleta de dados foi possível traçar um gráfico de temperatura em função do tempo para analisar o avanço da frente de combustão e o comportamento da evolução e perfil de temperatura ao longo do leito. Após a

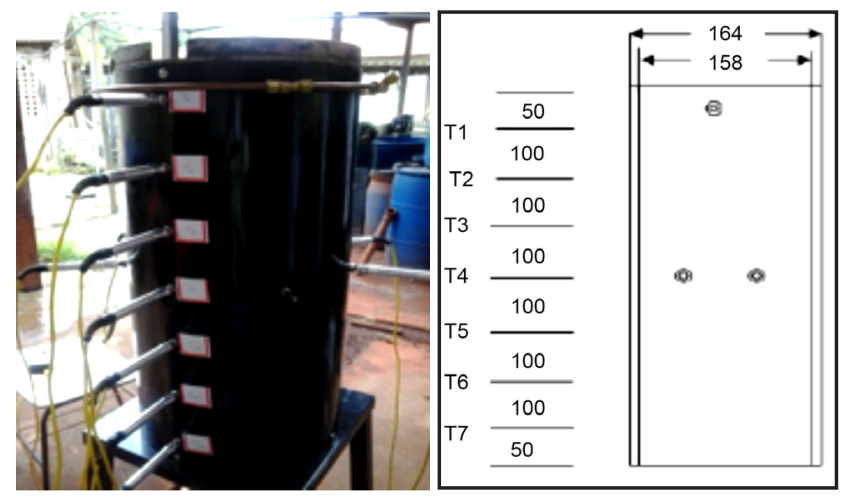

Figura 2: Distribuição dos termopares no reator. [Figure 2: Distribution of thermocouples in the reactor.] secagem da mistura com a corrente de alimentação do reator foi realizado o preenchimento do leito de sinterização. A velocidade do ar foi medida através do termoanemômetro CFM e ajustada para cada experimento com o auxílio de uma válvula globo, localizada próxima ao exaustor. Para dar início ao experimento um maçarico localizado na parte superior do reator foi conectado ao gás GLP que proporcionou uma chama regulável, utilizado para iniciar a combustão. Ao registrar $700{ }^{\circ} \mathrm{C}$ pelo primeiro termopar do reator, a chama regulável foi cessada de modo que, a partir desta temperatura o processo de combustão ocorreu de forma autônoma ao longo do leito reacional.

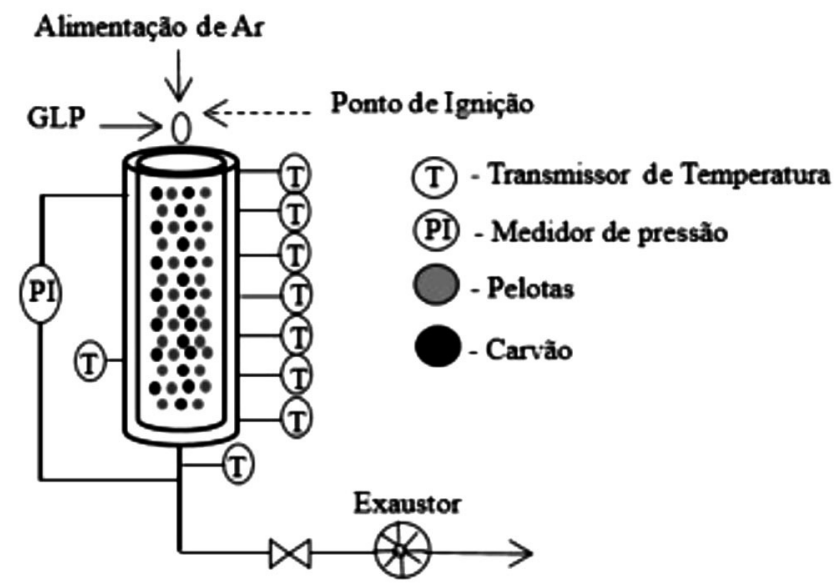

Figura 3: Desenho esquemático do dispositivo experimental. [Figure 3: Schematic of the experimental device.]

Agregado sintético produzido: após a sinterização no dispositivo experimental obteve-se o agregado sintético de tamanho variado, conforme pode ser observado na Fig. 4. No produto obtido fez-se uma britagem primária e posteriormente a distribuição granulométrica segundo a norma, determinação das propriedades cerâmicas [12], difração de raios $X$ e microscopia eletrônica de varredura (MEV), em superfície polida e submetida a ataque químico com ácido fluorídrico, solução $10 \%$.

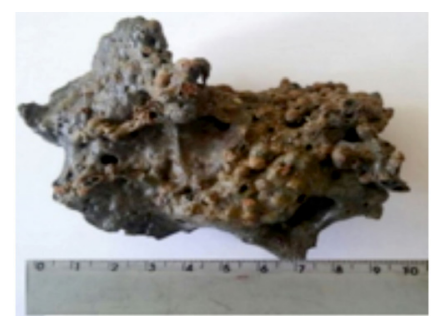

a)

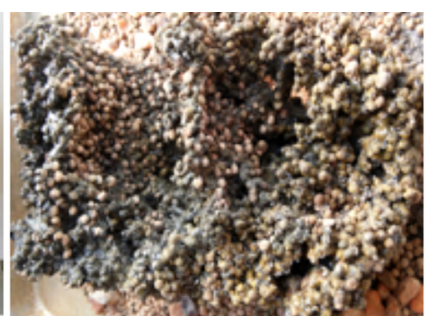

b)
Figura 4: Agregado sintético fabricado.

[Figure 4: Synthetic produced aggregate.]

\section{RESULTADOS E DISCUSSÃO}

Caracterização das matérias-primas: a Fig. 5 mostra o difratograma de raios $X$ e a curva de distribuição granulométrica da cinza volante. Conforme difratograma, 

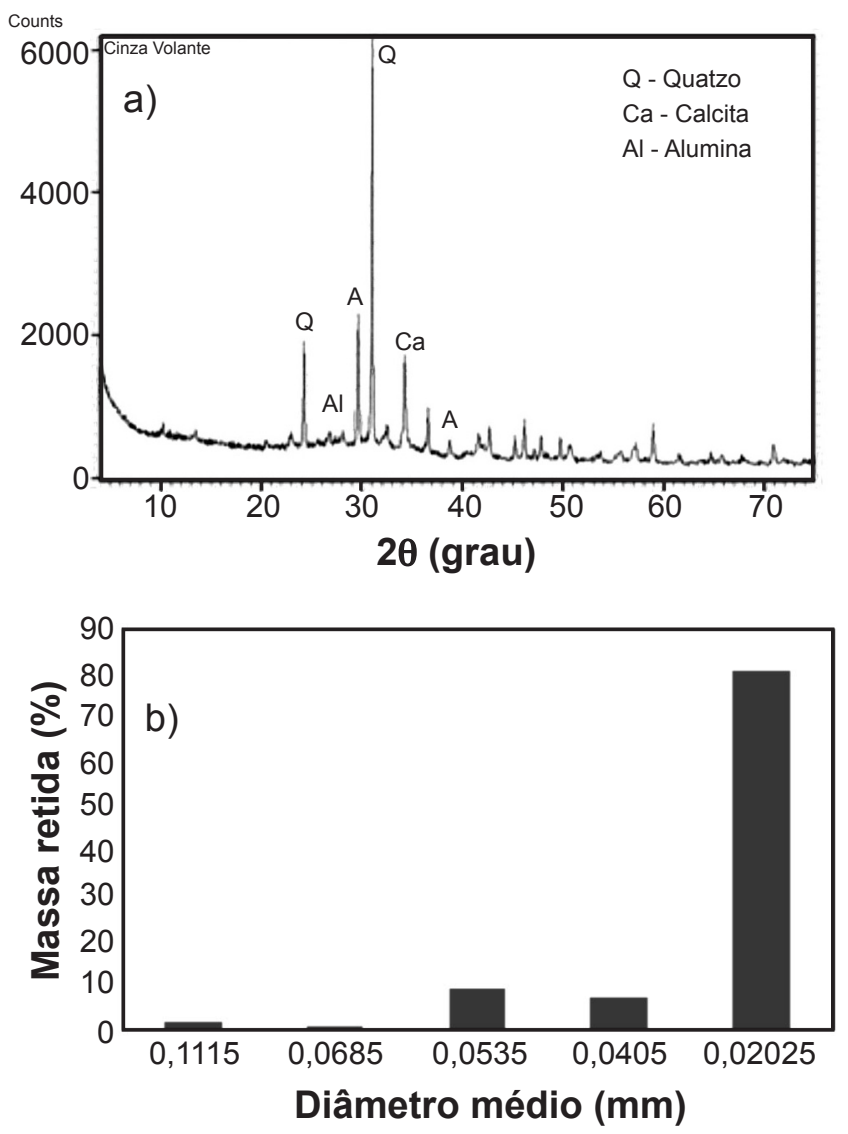

Figura 5: Difratograma de raios X (a) e distribuição granulométrica (b) da cinza.

[Figure 5: X-ray diffraction pattern (a) and particle size distribution (b) of fly ash.]

existiu a presença das fases calcita, quartzo e alumina. A presença da calcita deveu-se ao processo de dessulfuração interna durante a combustão do carvão mineral, onde calcário $\left(\mathrm{CaCO}_{3}\right)$ foi inserido para reduzir as emissões de gases como o enxofre $\left(\mathrm{SO}_{2}\right)$ na atmosfera [13]; a formação de quartzo foi justificada pela presença de sílica cristalina em temperaturas superior a $500{ }^{\circ} \mathrm{C}$, segundo o diagrama de fases [14]. Outro aspecto que justificou tanto a presença de quartzo $\left(\mathrm{SiO}_{2}\right)$ quanto a de alumina $\left(\mathrm{Al}_{2} \mathrm{O}_{3}\right)$ foi vinculado ao fato de que estas fases, presentes originalmente no carvão, se mantiveram durante todo o processo, uma vez que a temperatura de processamento de produção de carvão, normalmente inferior a $1000{ }^{\circ} \mathrm{C}$, não foi suficiente para transformar essas estruturas [14]. No que se refere à distribuição granulométrica da cinza volante, as partículas do material apresentaram diâmetro médio de $0,01087 \mathrm{~mm}$. Segundo normatização [15], a cinza volante foi classificada como material pozolânico de classe $\mathrm{C}$ em função de ser proveniente da queima de carvão mineral. No que se refere às concentrações de $\mathrm{SiO}_{2}, \mathrm{Al}_{2} \mathrm{O}_{3}$ e $\mathrm{Fe}_{2} \mathrm{O}_{3}$, a soma dos teores destes óxidos (Tabela II) superior a 50\% caracterizou este resíduo com propriedades cimentantes e pozolânicas [16].

A Fig. 6 apresenta o difratograma de raios $X$ da argila e sua distribuição granulométrica. Constatou-se a presença de fases mineralógicas típicas de argilas cauliníticas evidenciando a presença de caulinita e quartzo. Observouse na análise granulométrica que $98,17 \%$ do material tiveram tamanho de partícula inferior passante em malha de 400 mesh $(0,037 \mathrm{~mm})$ em peneira Tyler com diâmetro médio de $0,0210 \mathrm{~mm}$. A Tabela III apresenta resultados da análise química da argila com composição típica de argila caulinítica, por conta do elevado teor relativo de alumina $\left(\mathrm{Al}_{2} \mathrm{O}_{3}\right)$, baixo percentual de sílica $\left(\mathrm{SiO}_{2}\right)$ e baixo teor de fundentes alcalinos $\left(\mathrm{K}_{2} \mathrm{O}+\mathrm{Na}_{2} \mathrm{O}\right)$ [17].

A caracterização do carvão vegetal, com relação à
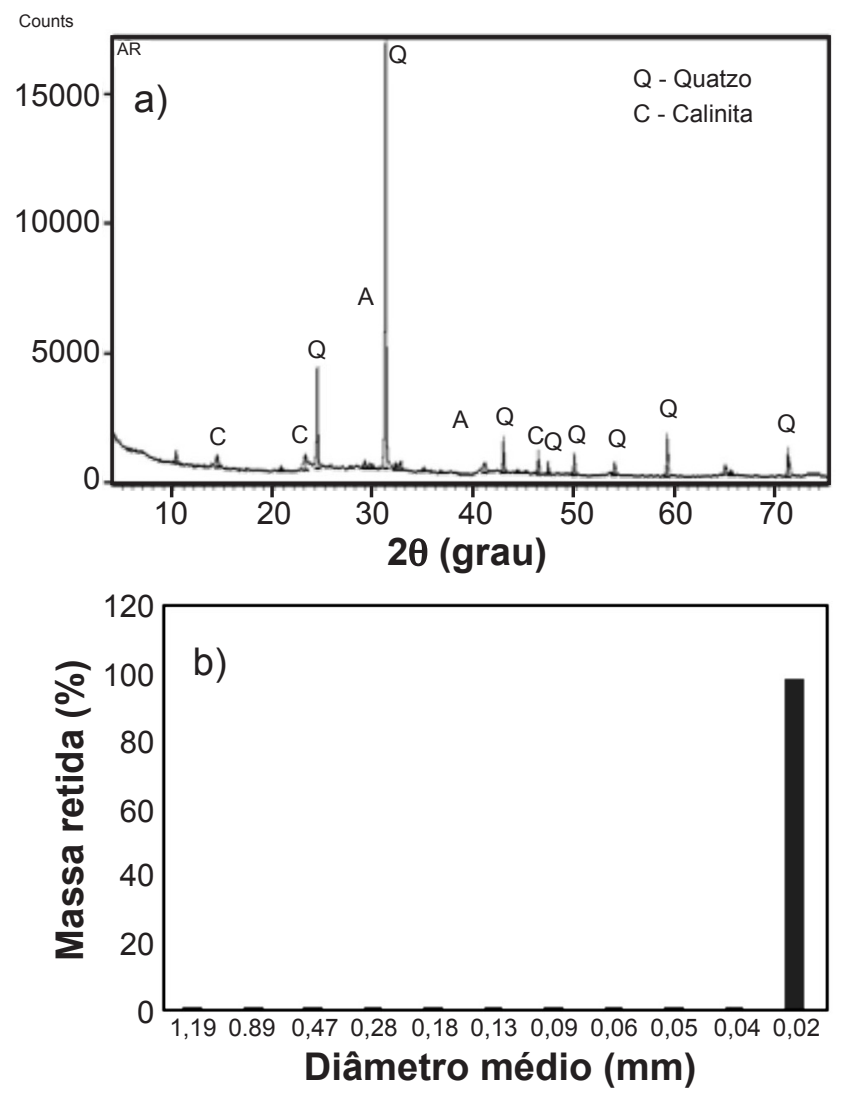

Figura 6: Difratograma de raios X (a) e distribuição granulométrica (b) da argila.

[Figure 6:X-ray diffraction pattern (a) and particle size distribution (b) of clay.]

Tabela II - Composição química (\% em massa) da cinza volante.

[Table II - Chemical composition (wt\%) of fly ash.]

\begin{tabular}{ccccccccccc}
\hline $\mathrm{Na}_{2} \mathrm{O}$ & $\mathrm{MgO}$ & $\mathrm{Al}_{2} \mathrm{O}_{3}$ & $\mathrm{SiO}_{2}$ & $\mathrm{P}_{2} \mathrm{O}_{5}$ & $\mathrm{~K}_{2} \mathrm{O}$ & $\mathrm{CaO}$ & $\mathrm{TiO}_{2}$ & $\mathrm{MnO}$ & $\mathrm{Fe}_{2} \mathrm{O}_{3}$ & $\mathrm{SO}_{3}$ \\
\hline 0,941 & 1,836 & 16,399 & 42,53 & 0,194 & 1,61 & 19,005 & 0,897 & 0,073 & 7,081 & 9,208 \\
\hline
\end{tabular}


Tabela III - Composição química (\% em massa) da argila.

[Table III - Chemical composition (wt\%) of clay.]

\begin{tabular}{ccccccccc}
\hline $\mathrm{SiO}_{2}$ & $\mathrm{Al}_{2} \mathrm{O}_{3}$ & $\mathrm{Fe}_{2} \mathrm{O}_{3}$ & $\mathrm{TiO}_{2}$ & $\mathrm{CaO}$ & $\mathrm{MgO}$ & $\mathrm{K}_{2} \mathrm{O}$ & $\mathrm{Na}_{2} \mathrm{O}$ & Perda ao fogo \\
\hline 48,18 & 19,56 & 9,38 & 2,02 & 0,73 & 1,24 & 2,79 & 1,6 & 14,50 \\
\hline
\end{tabular}

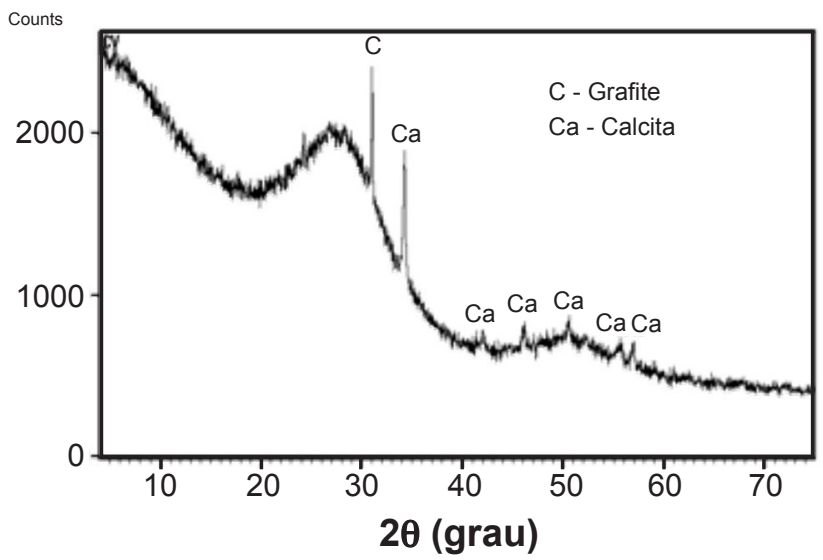

Figura 7: Difratograma de raios X do carvão vegetal. [Figure 7: X-ray diffraction pattern of charcoal.]

Tabela IV - Resultados da análise imediata do carvão vegetal. [Table IV - Results of immediate analysis of charcoal.]

\begin{tabular}{cccc}
\hline$\% \mathrm{U}$ & $\% \mathrm{MV}$ & $\% \mathrm{Z}$ & $\% \mathrm{CF}$ \\
\hline 4,71 & 15,24 & 19,48 & 65,28 \\
\hline
\end{tabular}

difração de raios X (Fig. 7), evidenciou a formação das fases grafita e calcita; a presença da primeira fase foi relacionada com a constituição característica da matéria-prima enquanto que a presença de calcita foi relacionada ao processo de fabricação do carvão apresentando-se como contaminante neste material. Quanto à análise imediata do carvão referente à determinação do teor de umidade $(\% \mathrm{U})$, matérias voláteis (\%MV), cinzas (\%Z) e carbono fixo (\%CF), o carvão apresentou elevado teor de carbono como pode ser observado na Tabela IV.

Caracterização das pelotas e do carvão granulado: a Fig. 8 mostra as curvas de distribuição de tamanho de partículas para as pelotas (Fig. 8a) e carvão de preenchimento do leito (Fig. 8b). O diâmetro médio de partícula destes materiais foram $5,13 \mathrm{~mm}$ e $1,95 \mathrm{~mm}$, respectivamente, garantindo de modo satisfatório a porosidade do leito para permitir o fluxo do ar e a consequente propagação da frente de combustão. A Tabela V apresenta os resultados da densidade aparente (DA) para pelotas, carvão de preenchimento do leito e mistura de alimentação do reator. A densidade aparente da mistura apresentou um valor que pode ser considerado próximo ao obtido para o carvão vegetal, fato que foi explicado em função do leito ser preenchido com $80 \%$ de carvão vegetal, o que favoreceu o empacotamento do leito de tal forma que o fluxo do ar percorresse os espaços vazios que estavam entre os grãos da mistura, permitindo que a combustão ocorresse de forma gradativa, garantindo o processo de sinterização
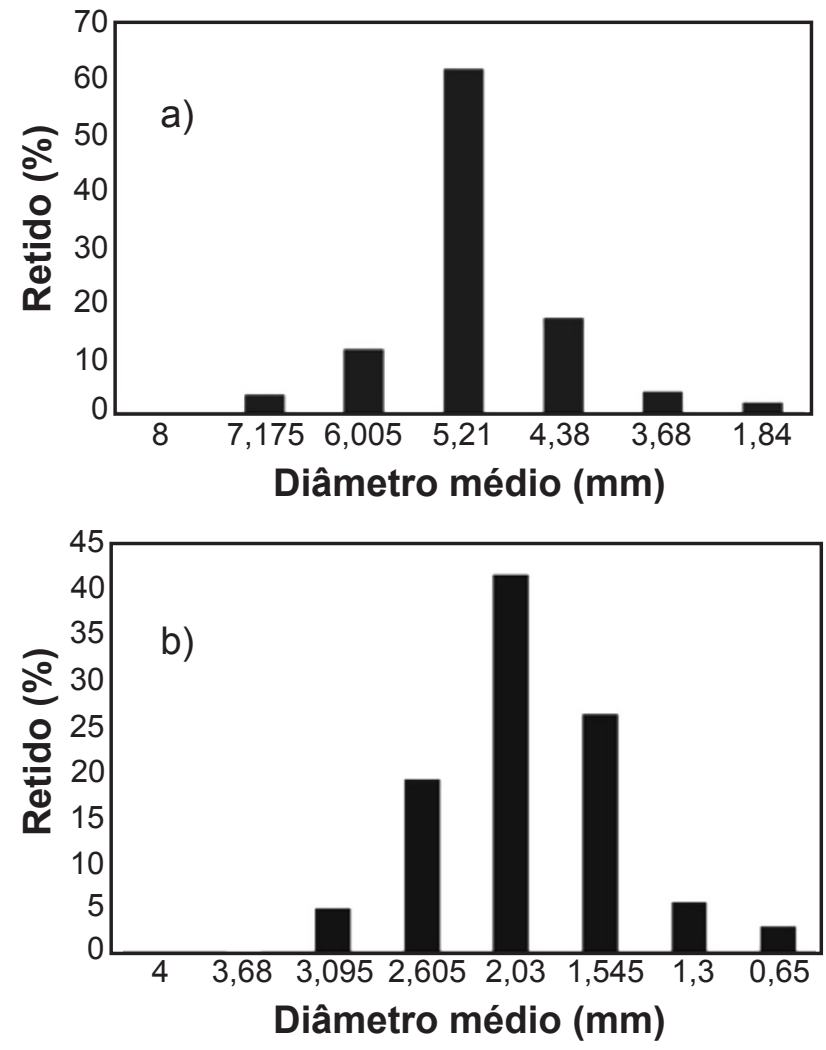

Figura 8: Distribuição de tamanho de partículas das pelotas (a) e do carvão de preenchimento do leito (b).

[Figure 8: Particle size distribution of pellets (a) and the coal for bed filler $(b)$.]

Tabela V - Densidade aparente dos materiais classificados.

[Table V-Bulk density of the used materials.]

\begin{tabular}{ccc}
\hline \multirow{2}{*}{ Material classificado } & \multicolumn{2}{c}{$\mathrm{D}_{\mathrm{A}}\left(\mathrm{g} \cdot \mathrm{cm}^{-3}\right)$} \\
& Experimento 1 & Experimento 2 \\
\hline Carvão vegetal & 0,22 & 0,22 \\
Pelota & 0,59 & 0,57 \\
Mistura (pelota e & 0,32 & 0,32 \\
carvão vegetal) & & \\
\hline
\end{tabular}

do material.

Evolução da temperatura: as Figs. 9a e 9b apresentam a evolução da temperatura para o Experimento 1 com 0,68 m.s. $\mathrm{s}^{-1}$ e Experimento $2 \mathrm{com} 1,26 \mathrm{~m} \cdot \mathrm{s}^{-1}$ de velocidade de sucção do ar, respectivamente. As curvas apresentaram aspectos semelhantes, em que a temperatura registrada em cada termopar atingiu um valor máximo seguido de uma 

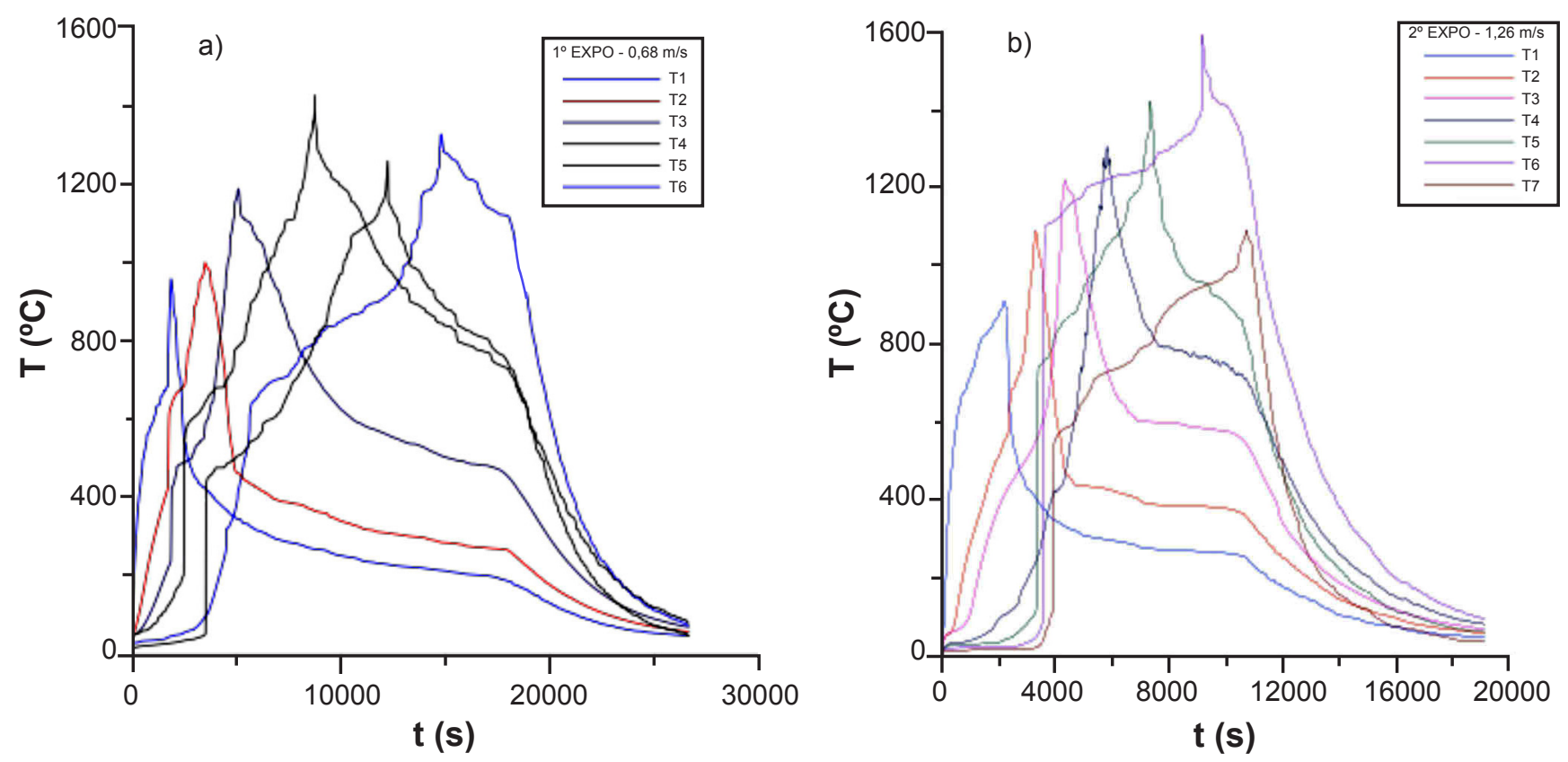

Figura 9: Evolução da temperatura para o Experimento 1 (a) e Experimento 2 (b).

[Figure 9: Temperature evolution for Experiment 1 (a) and Experiment 2 (b).]
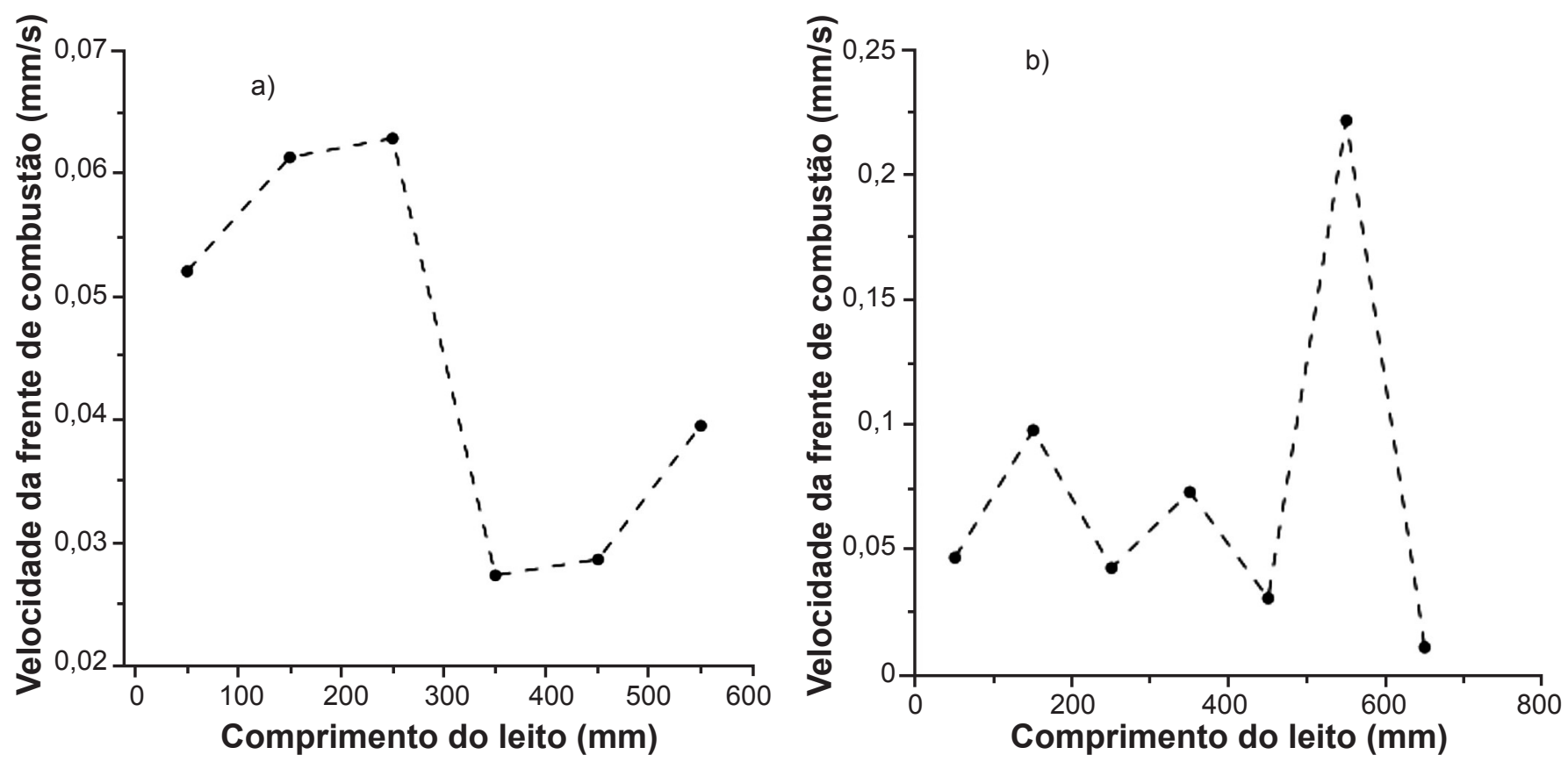

Figura 10: Velocidade da frente de combustão para o Experimento 1 (a) e Experimento 2 (b).

[Figure 10: Front burning rate for Experiment 1 (a) and Experiment 2 (b).]

redução gradual, caracterizando a evolução da temperatura ao longo do leito reacional. Para o Experimento 1, verificouse que as curvas tiveram um comportamento mais suave do que o Experimento 2. Atribuiu-se tal fato à baixa velocidade de sucção do ar do Experimento 1 quando comparado ao Experimento 2. Observou-se também que a interação da transferência de calor e massa entre as fases gás e sólido para reatores de leito fixo gerou um maior acúmulo de energia na forma de calor à medida que ocorreu o avanço da frente de combustão [18]. Esta interação ocorreu de modo mais intenso na região de 350, 450 e $550 \mathrm{~mm}$ a partir da alimentação do reator para o Experimento 1 e ao longo de todo o leito reacional para o Experimento 2.

Velocidade da frente de combustão: as Figs. 10a e 10b apresentam a velocidade da frente de combustão para o Experimento 1 e Experimento 2, em função do comprimento do leito, respectivamente. Observou-se que no início do experimento, houve a formação de caminhos preferenciais, 
onde a percolação do ar foi facilitada pela porosidade do leito e velocidade de sucção do ar fazendo com que a velocidade da frente de combustão aumentasse. No entanto, à medida que este leito foi consumido, um novo empacotamento foi criado com uma diminuição significativa de porosidade resultando em uma maior dificuldade para percolação do ar e consequente diminuição da velocidade da frente de combustão [19], onde se conceitua o fenômeno de sinterização como um processo onde partículas do material se ligam por difusão no estado sólido transformando um material poroso e compacto em um produto resistente e denso. No término do Experimento 1, verificou-se aumento da velocidade da frente de combustão, pois nesta região o leito reacional foi em grande parte sinterizado aderindo à parede interna do reator permitindo uma maior passagem de ar resultando em aumento da velocidade da frente de combustão, o que não se constatou ao término do Experimento 2, possivelmente devido à obstrução por material sinterizado concentrado no fundo do reator. Quando comparados os dois experimentos, verificou-se que a velocidade da frente de combustão do Experimento 1 apresentou comportamento mais suave do que o Experimento 2. Atribuiu-se tal fato à baixa velocidade de sucção do ar no Experimento $1\left(0,68 \mathrm{~m} \cdot \mathrm{s}^{-1}\right)$ comparada à velocidade do Experimento $2\left(1,26 \mathrm{~m} \cdot \mathrm{s}^{-1}\right)$.

Análises no agregado sintético: as Figs. 11a e 11b apresentam os resultados da análise granulométrica dos agregados produzidos no Experimento $1 \mathrm{e} 2$, respectivamente. Verificou-se que o diâmetro médio de partícula do material sinterizado foi de 7,55 e $7,20 \mathrm{~mm}$, respectivamente,
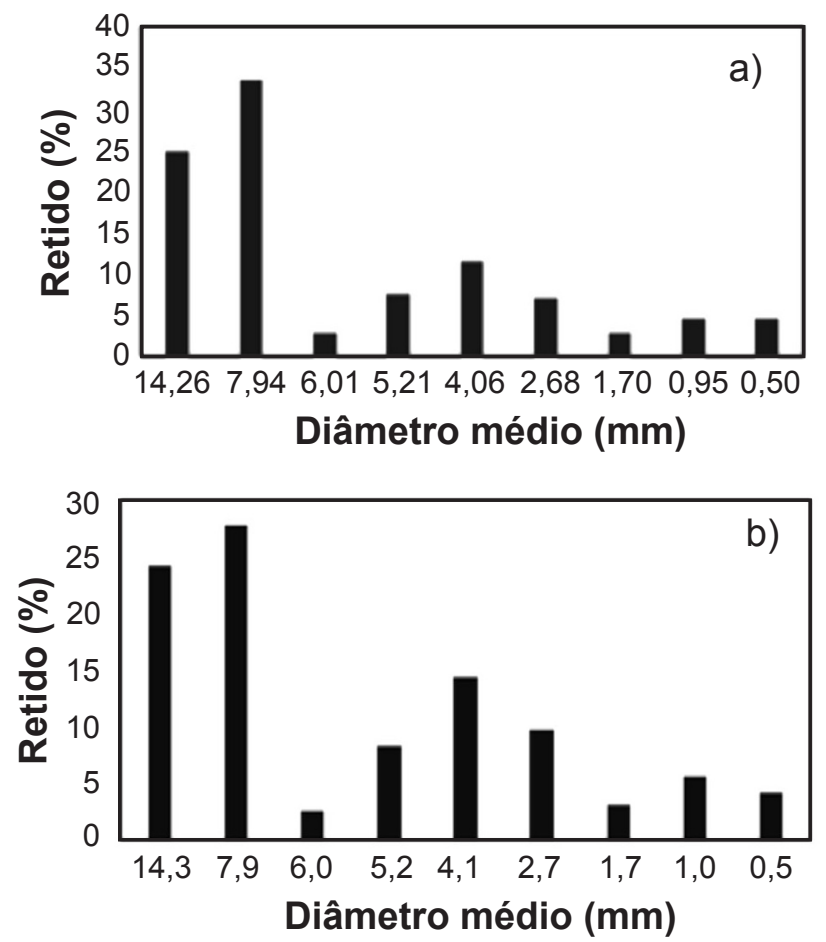

Figura 11: Distribuição granulométrica do material sinterizado para o Experimento 1 (a) e Experimento 2 (b).

[Figure 11: Particle size distribution of the sintered material for Experiment 1 (a) and Experiment 2 (b).]
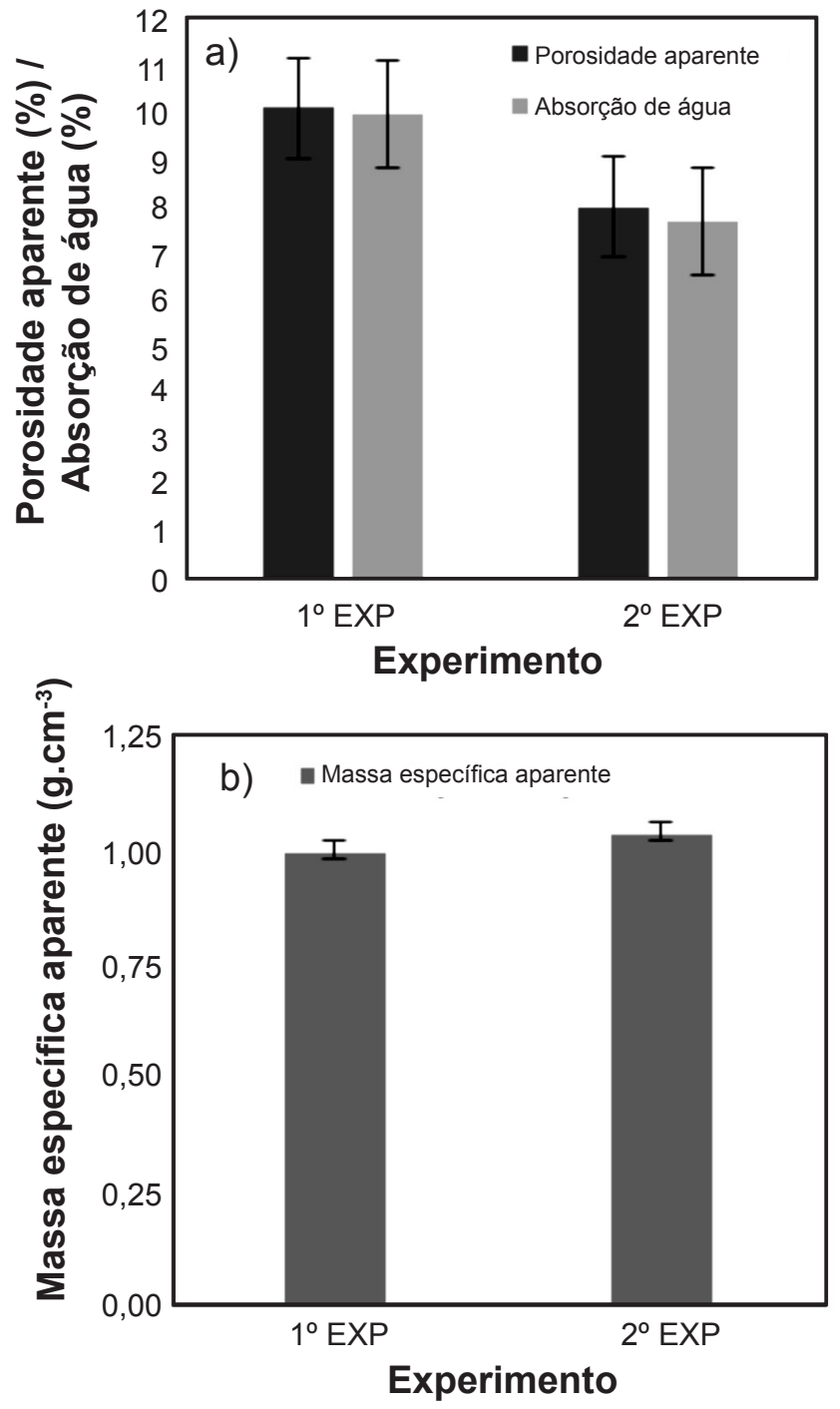

Figura 12: Medidas da porosidade aparente e absorção de água (a) e massa específica aparente (b) dos agregados.

[Figure 12: Measurements of apparent porosity and water absorption (a) and bulk density of aggregates.]

caracterizando esses materiais quanto ao seu tamanho como agregado graúdo conforme normatização brasileira [8].

A Fig. 12a apresenta as medidas da porosidade aparente e absorção de água que em conjunto com a massa específica aparente (Fig. 12b) correspondem às propriedades cerâmicas obtidas nos agregados de cada experimento. Os resultados obtidos permitiram classificar o material produzido como agregado leve, pois a massa específica aparente de cada experimento apresentou valor abaixo de $2 \mathrm{~g} . \mathrm{cm}^{-3}$ [20]. Comparando os experimentos observa-se que o Experimento 1 apresentou menor valor de massa específica aparente em função de sua elevada porosidade, justificado pelo menor grau de sinterização neste experimento comparado ao Experimento 2. Os resultados obtidos evidenciaram que com o aumento da porosidade aparente houve aumento gradativo da absorção de água e consequente diminuição da massa específica aparente, fato que pode ser explicado quando se refere à produção de agregado leve por meio de 
sinterização [12]. Como consequência dos elevados valores de porosidade, esses materiais apresentaram significativa capacidade de absorção de água, poros abertos, além de não possuir recobrimento, fato este que permitiu inferir que houve uma redução na formação da fase amorfa do material.

As Fig. 13a e 13b apresenta os difratogramas de raios $\mathrm{X}$ dos materiais obtidos a partir do Experimento 1 e 2, respectivamente. Os resultados mostraram a presença de quatro fases mineralógicas: quartzo $\left(\mathrm{SiO}_{2}\right)$, mulita $\left(3 \mathrm{Al}_{2} \mathrm{O}_{3} \cdot 2 \mathrm{SiO}_{2}\right)$, anortita $\left(\mathrm{CaAl}_{2} \mathrm{Si}_{2} \mathrm{O}_{8}\right)$ e esseneita $\left(\mathrm{CaFe}^{3+} \mathrm{AlSiO}_{6}\right)$. A formação de quartzo pode ser justificada pela presença de sílica em temperaturas acima de $500{ }^{\circ} \mathrm{C}$. A presença de mulita foi relacionada à presença de sílicoaluminatos em temperaturas acima de $1100{ }^{\circ} \mathrm{C}$ [5]. A constatação da presença das fases anortita e esseneita foi vinculada também à presença de sílico-aluminatos, além de cálcio e ferro disponível em temperaturas favoráveis à formação destas fases, superiores a $1000{ }^{\circ} \mathrm{C}$. Observouse que independente da variação de velocidade de sucção do ar para cada experimento, qualitativamente houve a formação das mesmas fases mineralógicas comprovando que estas foram funções da temperatura de sinterização e dos constituintes químicos da mistura.

No que se refere à microestrutura, as Figs. 14a e 14b apresentam as micrografias de MEV dos agregados
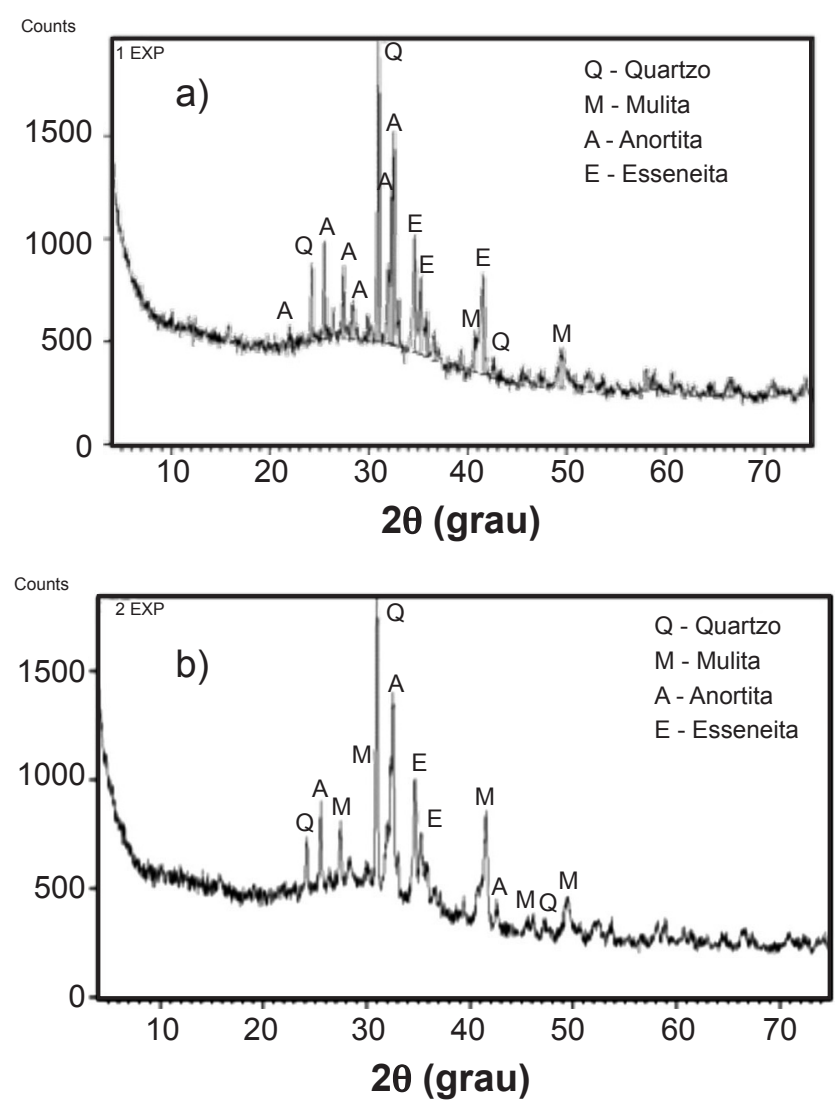

Figura 13: Difratogramas de raios $\mathrm{X}$ dos agregados para $\mathrm{O}$ Experimento 1 (a) e Experimento 2 (b).

[Figure 13:X-ray diffraction patterns of aggregates for Experiment 1 (a) and Experiment $2(b)$.
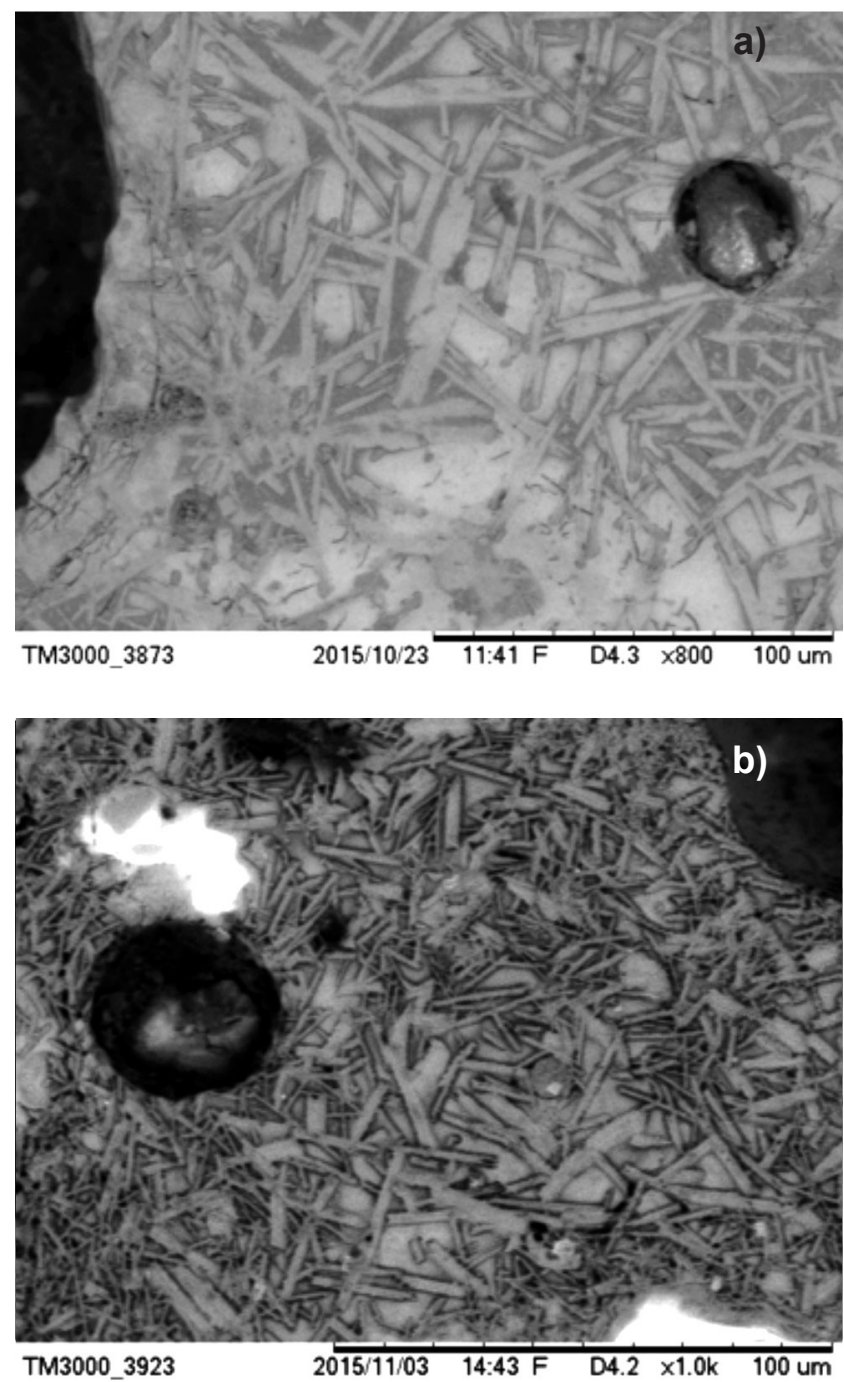

Figura 14: Micrografias obtidas por microscopia eletrônica de varredura dos agregados para o Experimento 1 (a) e Experimento 2 (b).

[Figure 14: SEM micrographs of aggregates for Experiment 1 (a) and Experiment $2(b)$.

preparados nos Experimentos 1 e 2, respectivamente. As micrografias comprovaram a formação de mulita primária e secundária de morfologia acicular. Esta formação foi vinculada à eficiência das reações de estado sólido, que por sua vez foram funções de elevadas temperaturas e composição da mistura, uma vez que estas foram formadas majoritariamente por sílico-aluminatos [21].Além dos fatores citados anteriormente, a presença de cátions vitrificantes (K, $\mathrm{Ca}, \mathrm{Fe}$ e $\mathrm{Na}$ ) na mistura sinterizada facilitaram a nucleação e crescimento de mulita a partir da redução de viscosidade da fase amorfa [22]. Quando comparados os dois experimentos, constatou-se que a variação de velocidade de sucção do ar não interferiu na formação de mulita. No entanto, observou-se que no agregado do Experimento 1, em que a velocidade de sucção foi menor, uma estrutura constituída em sua maioria de mulitas primárias (na forma de cristais lamelares) e nucleação e crescimento de mulita secundária a partir da estrutura de mulitas primárias em algumas 
regiões. No que se refere ao agregado do Experimento 2, verificou-se a presença de uma estrutura constituída de partículas de quartzo, regiões de nucleação e crescimento de mulitas secundárias (monocristais na forma de agulhas) a partir, também, de morfologia primária, mulitas secundárias totalmente desenvolvidas e mulitas primárias.

\section{CONCLUSÕES}

O reator vertical de leito fixo permitiu um satisfatório processo de sinterização para obtenção do agregado sintético, pois de acordo com as evoluções e perfis de temperatura, $\mathrm{o}$ processo de sinterização ocorreu em temperaturas elevadas, acima de $1000{ }^{\circ} \mathrm{C}$, em todos os experimentos, fato que garantiu a eficiência das reações de estado sólido. A granulometria definida tanto das pelotas quanto do carvão vegetal permitiu que o leito do reator fosse suficientemente poroso para permitir o fluxo do ar e a propagação da frente de combustão, e assim garantir a sinterização das pelotas, durante as reações de combustão do carvão vegetal. Através desses dados concluiu-se que é possível controlar a taxa de avanço da interface de calor, temperatura e tempo de queima, variando-se a velocidade de sucção do ar. Materiais ricos em sílica e alumina como cinza volante e argila, sinterizados a partir de $1100{ }^{\circ} \mathrm{C}$ reagiram formando mulita, composto estável, responsável pela característica de resistência mecânica ao material sinterizado. Constatou-se que a variação de velocidade de sucção de ar não interferiu qualitativamente na formação de mulita comprovada nas análises de microscopia eletrônica de varredura e difração de raios X. Em conjunto com os dados obtidos para massa específica aparente, os valores elevados de porosidade e absorção aparente formaram resultados característicos do processo de sinterização de agregado leve. Acrescido a isso, os valores de porosidade aparente mostraram que a formação de fase amorfa (vidro) foi reduzida o que de certa forma é interessante para o agregado pela capacidade de aderência ao concreto. Segundo a normatização brasileira os agregados sintéticos produzidos, em ambos os experimentos, podem ser classificados como agregado graúdo, pois apresentaram diâmetro médio de partícula superior a 4,75 $\mathrm{mm}$. O material produzido tem potencial para substituir agregados naturais, com controle das propriedades cerâmicas e resistência mecânica, melhorando a qualidade do concreto.

\section{AGRADECIMENTOS}

Os autores agradecem a CAPES, CNPQ, HYDRO Alunorte e Universidade Federal do Pará pelo suporte fornecido a este trabalho.

\section{REFERÊNCIAS}

[1] F.A.N. Silva, S.N. Monteiro, C.M.F. Vieira, M.F.S. Lopes, Cerâmica 52, 321 (2006) 15.

[2] F. Chies, N.I.W. Silva, O. Zwosnok, Rocha, Coleção Habitare 4 (2003) 218.

[3] L.M. Calarge, N.I.W. Silva, F. Chies, O. Zwosnok, Gerenciamento de Resíduos Sólidos e Certificação Ambiental 1 (2000) 130.

[4] S. Sabedot, M.G. Sundstron, S.C. de Böer, C.H. Sampaio, R.G.O. Dias, C.G. Ramos, III Congr. Bras. Carvão Mineral, Gramado, RS (2011).

[5] E.M. Cabral, R.J. Sá, R.K. Vieira, R.P. Vasconcelos, Cerâmica 54, 332 (2008) 404.

[6] A. Paprocki, A.M. Cardoso, R.A. Ligavue, XVIII Congr. Bras. Eng. Ciên. Mater., Porto de Galinhas, PE (2008) 676.

[7] M.F. Martins, S. Salvador, J.F. Thovert, G. Debenest, Fuel 89 (2010) 133.

[8] ABNT - Assoc. Brasil. Normas Téc., NBR 7211, Rio de Janeiro (1983).

[9] ABNT - Assoc. Brasil. Normas Téc., NBR 8112, Rio de Janeiro (1986).

[10] A.O. Melo, E.N. Macêdo, M.F. Martins, D. Quaresma, J.A.S. Souza, A. Neves, $23^{\text {rd }}$ ABCM Int. Congr. Mech. Eng., Rio de Janeiro, RJ (2015).

[11] EMBRAPA, Manual de métodos de análise de solos, $2^{\mathrm{a}}$ ed., Rio de Janeiro (1997).

[12] P.S. Santos, Ciência e tecnologia de argilas, $2^{\mathrm{a}}$ ed., Edgard Blücher, S. Paulo (1989) 408.

[13] J.S. Siqueira, A.G. Souza, J.A.S. Souza, Cerâmica 58, 346 (2012) 257.

[14] C.A.F. Rocha Jr., S.C.A. Santos, C.A.G. Souza, R.S. Angélica, R.F. Neves, Cerâmica 58, 345 (2012) 43.

[15] ABNT - Assoc. Brasil. Normas Téc., NBR 12653, Rio de Janeiro (2014).

[16] Am. Soc. Testing Materials, ASTM C618, Philadelphia (1978).

[17] C.M.F. Vieira, R.M. Pinheiro, Cerâmica 57, 343 (2011) 319.

[18] M.L. Hobbs, P.T. Radulovic, L.D. Smoot, Prog. Energy Combust. Sci. 19 (1993) 505.

[19] F.H. Norton, Introdução a tecnologia cerâmica, S. Paulo, Edgard Blucher (1973).

[20] ABNT - Assoc. Brasil. Normas Téc., NBR 9935, Rio de Janeiro (2011).

[21] G.W. Brindley, M. Nakahira, Nature 181 (1958) 1333.

[22] H. Mörtel, C. Boubacar, Deutschen Keramischen Gesellschaft 54 (1977) 264.

(Rec. 05/02/2016, Rev. 19/04/2016, 13/07/2016, Ac. 27/09/2016) 